

Л. А. Петров, Предельное поведение некоторых случайных блужданий на строгих разбиениях, УМН, 2009, том 64, выпуск 6, 177-178

DOI: https://doi.org/10.4213/rm9318

Использование Общероссийского математического портала Math-Net.Ru подразумевает, что вы прочитали и согласны с пользовательским соглашением http://www . mathnet.ru/rus/agreement

Параметры загрузки:

IP: 54.89 .56 .158

26 апреля 2023 г., 18:35:42

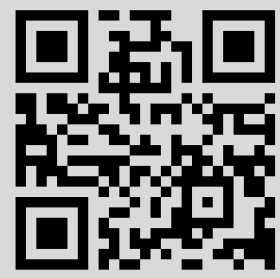




\section{Предельное поведение некоторых случайных блужданий на строгих разбиениях}

\section{Л. А. Петров}

В статье строится однопараметрическое семейство марковских процессов с непрерывным временем $\mathbf{X}_{\alpha}(t), t \geqslant 0$, на бесконечномерном упорядоченном симплексе

$$
\Omega_{+}:=\left\{\mathrm{x}=\left(\mathrm{x}_{1}, \mathrm{x}_{2}, \ldots\right): \mathrm{x}_{1} \geqslant \mathrm{x}_{2} \geqslant \cdots \geqslant 0, \sum_{i=1}^{\infty} \mathrm{x}_{i} \leqslant 1\right\}
$$

и изучаются некоторые его свойства. Здесь $\alpha$ - параметр, который принимает действительные положительные значения.

Сначала опишем процесс $\mathbf{X}_{\alpha}(t)$ в терминах его генератора.

В топологии покоординатной сходимости $\Omega_{+}$является метризуемым сепарабельным компактом. Обозначим через $C\left(\Omega_{+}\right)$алгебру непрерывных действительнозначных функций на $\Omega_{+}$с поточечными операциями и равномерной нормой. Положим $\mathrm{q}_{k}(\mathrm{x}):=\sum_{i=1}^{\infty} \mathrm{x}_{i}^{k+1}, k=1,2, \ldots, \mathrm{x} \in \Omega_{+}$. Функции $\mathrm{q}_{k}$ являются непрерывными и алгебраически независимыми. Любой бесконечный набор функций вида $\left\{\mathbf{q}_{k_{1}}, \mathbf{q}_{k_{2}}, \ldots\right\}$ разделяет точки пространства $\Omega_{+}$. Следуя работе [1], будем называть функции $\mathrm{q}_{k}$, $k=1,2, \ldots$, моментными координатами на пространстве $\Omega_{+}$. Пусть $\mathcal{F}$ - коммутативная алгебра с единицей, свободно порожденная моментными координатами с четными номерами: $\mathcal{F}=\mathbb{R}\left[\mathbf{q}_{2}, \mathbf{q}_{4}, \mathbf{q}_{6}, \ldots\right]$. Зафиксируем произвольное значение параметра $\alpha \in(0,+\infty)$. Определим формальный дифференциальный оператор второго порядка $A$ в алгебре $\mathcal{F}$ следующим образом:

$$
\begin{aligned}
A:= & \sum_{i, j=1}^{\infty}(2 i+1)(2 j+1)\left(\mathbf{q}_{2 i+2 j}-\mathbf{q}_{2 i} \mathbf{q}_{2 j}\right) \frac{\partial^{2}}{\partial \mathbf{q}_{2 i} \partial \mathbf{q}_{2 j}} \\
& +2 \sum_{i, j=1}^{\infty}(2 i+2 j-1) \mathbf{q}_{2 i-2} \mathbf{q}_{2 j-2} \frac{\partial}{\partial \mathbf{q}_{2 i+2 j-2}}-\sum_{i=1}^{\infty}(2 i+1)\left(2 i+\frac{\alpha}{2}\right) \mathbf{q}_{2 i} \frac{\partial}{\partial \mathbf{q}_{2 i}}
\end{aligned}
$$

где для удобства полагаем $\mathrm{q}_{0}:=1$.

Teорема. 1) Оператор $A$ замыкаем в $C\left(\Omega_{+}\right)$. 2) Его замыкание $\bar{A}$ порождает дифбузионный прочесс $\left\{\mathbf{X}_{\alpha}(t)\right\}_{t \geqslant 0}$ на $\Omega_{+}$(под дифбузионным прочессом мы понимаем строго марковский прочесс с непрерывными траекториями). 3) У прочесса $\mathbf{X}_{\alpha}(t)$ существует единственная инвариантная вероятностная мера $\mathrm{P}^{(\alpha)}$. Процесс обратим относительно этой меры. 4) Спектр генератора $\bar{A}$ имеет вид $\{0\} \cup\{-m(m+$ $\alpha / 2-1): m=2,3, \ldots\}$. Собственное значение 0 простое, а кратность $m$-го равна количеству разбиений числа $m$ в сумму нечетных слагаемых, больших единицы, т.е. числу решений уравнения $3 n_{3}+5 n_{5}+7 n_{7}+\cdots=m$ в неотрицательных челых числах.

Процесс $\mathbf{X}_{\alpha}(t)$ на $\Omega_{+}$строится как предел случайных блужданий с растущим (но конечным) числом состояний. При этом один шаг $n$-го блуждания соответствует малому интервалу времени порядка $n^{-2}$. Перейдем к описанию случайных блужданий.

Пространство состояний $n$-го блуждания есть множество $\mathbb{S}_{n}$ всех разбиений числа $n=0,1,2, \ldots$ в сумму различных натуральных слагаемых (так называемых строгих разбиений), или, что то же самое, множество всех сдвинутых диаграмм Юнга с $n$ клетками. Отождествление строгих разбиений со сдвинутыми диаграммами Юнга описано, например, в [2].

Случайные блуждания на $\mathbb{S}_{n}, n=1,2, \ldots$, строятся в рамках формализма, развитого в работах [1], [3] и др. В нашей конкретной ситуации также используются 
результаты работы [4]. Впервые в литературе подобные марковские цепи (на множестве всех разбиений) были рассмотрены в работе [5]. Следуя [1], будем называть наши блуждания марковскими иепями вверх/вниз. Формализм состоит в следующем. Для каждой борелевской вероятностной меры $\mathrm{P}$ на $\Omega_{+}$существует канонически с ней связанная последовательность вероятностных мер $M_{n}$ на $\mathbb{S}_{n}$. Она называется когерентным семейством мер и однозначно определяет меру Р. Также можно канонически задать последовательность $T_{n}$ марковских цепей вверх/вниз, где $T_{n}-$ соответствующий оператор в пространстве всех функций на $\mathbb{S}_{n}$. Обозначим это пространство через $\operatorname{Fun}\left(\mathbb{S}_{n}\right)$. Мера $M_{n}$ является инвариантной мерой для $T_{n}$; цепь эргодична и обратима относительно этой меры. Более подробно о построении мер $M_{n}$ и цепей $T_{n}$ см. в $[1 ; \S 1]$.

Найден предел цепей $T_{n}$ при $n \rightarrow \infty$ в специальном случае меры $\mathrm{P}$ на $\Omega_{+}$. Эта мера $\mathrm{P}=\mathrm{P}^{(\alpha)}$ (которая участвует в теореме) определяется по мультипликативному когерентному семейству мер $\left\{M_{n}^{\alpha}\right\}$, зависящему от параметра $\alpha \in(0,+\infty)$. Семейство $\left\{M_{n}^{\alpha}\right\}$ было введено А. М. Бородиным в работе [6]. Предел цепей $T_{n}$ и есть процесс $\mathbf{X}_{\alpha}(t)$ на $\Omega_{+}$.

Опишем, в каком смысле случайные блуждания $T_{n}$ сходятся к $\mathbf{X}_{\alpha}(t)$. Для каждого $n=1,2, \ldots$ рассмотрим вложение $\iota_{n}: \mathbb{S}_{n} \hookrightarrow \Omega_{+}, \iota_{n}(\lambda)=\left(\lambda_{1} / n, \lambda_{2} / n, \ldots\right) \in \Omega_{+}$, где $\lambda \in \mathbb{S}_{n}$. Пусть $\pi_{n}: C\left(\Omega_{+}\right) \rightarrow \operatorname{Fun}\left(\mathbb{S}_{n}\right)$ - соответствующая $\iota_{n}$ проекция пространств функций, т. е. $\left(\pi_{n} f\right)(\lambda):=f\left(\iota_{n}(\lambda)\right)$, где $f \in C\left(\Omega_{+}\right)$и $\lambda \in \mathbb{S}_{n}$. Путем алгебраических вычислений, которые и составляют основную работу по построению процесса, можно показать, что операторы $n^{2}\left(T_{n}-\mathbf{1}\right)$ сходятся к оператору $A$, определенному выше, в том смысле, что

$$
\lim _{n \rightarrow \infty} \sup _{\lambda \in \mathbb{S}_{n}}\left|\left(n^{2}\left(T_{n}-\mathbf{1}\right) \pi_{n} f\right)(\lambda)-\left(\pi_{n} A f\right)(\lambda)\right|=0 \quad \text { для всех } f \in \mathcal{F} .
$$

Из этого факта и общих теорем [7; теорема 5.3] и [8; гл. 1, теорема 6.5] об аппроксимации непрерывной полугруппы дискретными следует, что $T_{n}^{\left[n^{2} t\right]} \rightarrow T(t)$, или, более подробно,

$$
\lim _{n \rightarrow \infty} \sup _{\lambda \in \mathbb{S}_{n}}\left|\left(T_{n}^{\left[n^{2} t\right]} \pi_{n} f\right)(\lambda)-\left(\pi_{n} T(t) f\right)(\lambda)\right|=0 \quad \text { для всех } f \in C\left(\Omega_{+}\right)
$$

равномерно по $t \in\left[0, t_{0}\right]$ для всех $t_{0} \geqslant 0$, где $\{T(t)\}_{t \geqslant 0}-$ полугруппа процесса $\mathbf{X}_{\alpha}(t)$, порожденная оператором $\bar{A}$.

Из сходимости дискретных полугрупп $\left\{1, T_{n}, T_{n}^{2}, \ldots\right\}$ к непрерывной полугруппе $\{T(t)\}_{t \geqslant 0}$ можно получить все остальные утверждения нашей теоремы.

Автор выражает благодарность Г. Ольшанскому за постановку задачи и постоянное внимание к работе, а также В. Иванову за полезные обсуждения.

\section{Список литературы}

[1] A. Borodin, G. Olshanski, Probab. Theory Related Fields, 144:1-2 (2009), 281-318; arXiv: 0706.1034v1. [2] И. Макдональд, Симметрические функиии и многочлены Холла, Мир, М., 1985. [3] S. Kerov, A. Okounkov, G. Olshanski, Internat. Math. Res. Notices, 1998:4 (1998), 173-199. [4] В. Н. Иванов, Записки науч. сем. ПОМИ, 240 (1997), 115-135. [5] J. Fulman, Trans. Amer. Math. Soc., 357:2 (2005), 555-570. [6] А. М. Бородин, Записки науч. сем. ПОМИ, 240 (1997), 44-52. [7] H.F. Trotter, Pacific J. Math., 8 (1958), 887-919. [8] S. N. Ethier, T. G. Kurtz, Markov processes. Characterization and convergence, Wiley, New York, 1986.

Л. А. Петров (L. A. Petrov)

Институт проблем передачи информации им. А. А. Харкевича РАН

E-mail: lenia.petrov@gmail.com
Представлено Р. А. Минлосом Принято редколлегией 09.08.2009 